\title{
4.5G Capacity \& QoS Optimization: A New Proposal to Enhance the Principal KPIs of 5G/IoT
}

\author{
https://doi.org/10.3991/ijoe.v14i11.9256
}

\author{
Ahmed El Mahjoubi $\left.{ }^{\bowtie}\right)$, Tomader Mazri, Nabil Hmina \\ National School of Applied Sciences Kenitra, Morocco \\ elmahjoubi.ahmed@univ-ibntofail.ac.ma
}

\begin{abstract}
RF capacity management and optimization is an important part of the network performances of the future. Technically, $4.5 \mathrm{G} / 5 \mathrm{G}$ mobile network is expected to enable people-thing and thing-thing interconnections by combining communication technologies and networks. Our Lab has been actively promoting research on $4 \mathrm{G} \& 5 \mathrm{G}$ performances, in which the RF Capacity management issue is of great interest. New $4 \mathrm{G} / 5 \mathrm{G}$ tests put forward a lot of requirements for RF network performances in terms of KPIs, QoS, as well as user experience. Based on Morocco's current situation, this article first discusses the LTE cell resources monitoring and optimization, Furthermore, we will introduce LTE OMSTAR tool, and LTE cell resources optimization scenarios are summarized.
\end{abstract}

Keywords - LTE Capacity, RF Optimization, 5G, QoS, IoT.

\section{Introduction}

With the rapid development of smart terminals and services the impact of capacity problems on KPIs and User experience increased, therefore close monitoring for Key bottleneck of LTE resources is required to ensure better KPIs and user experience.

Capacity monitoring can be implemented using the following two methods: [1]

- Daily monitoring for prediction: Counters are used to indicate the load or usage of various types of resources on the LTE network. Thresholds for resource consumption are specified so that preventive measures such as reconfiguration and expansion can be taken to prevent network congestion when the consumption of a type of resource continually exceeds the threshold. [2]

- Problem-driven analysis: This method helps identify whether a problem indicated by counters is caused by network congestion through in-depth analysis. With this method, problems can be precisely located so that users can work out a proper network optimization and expansion solution.

LTE Resources are: 

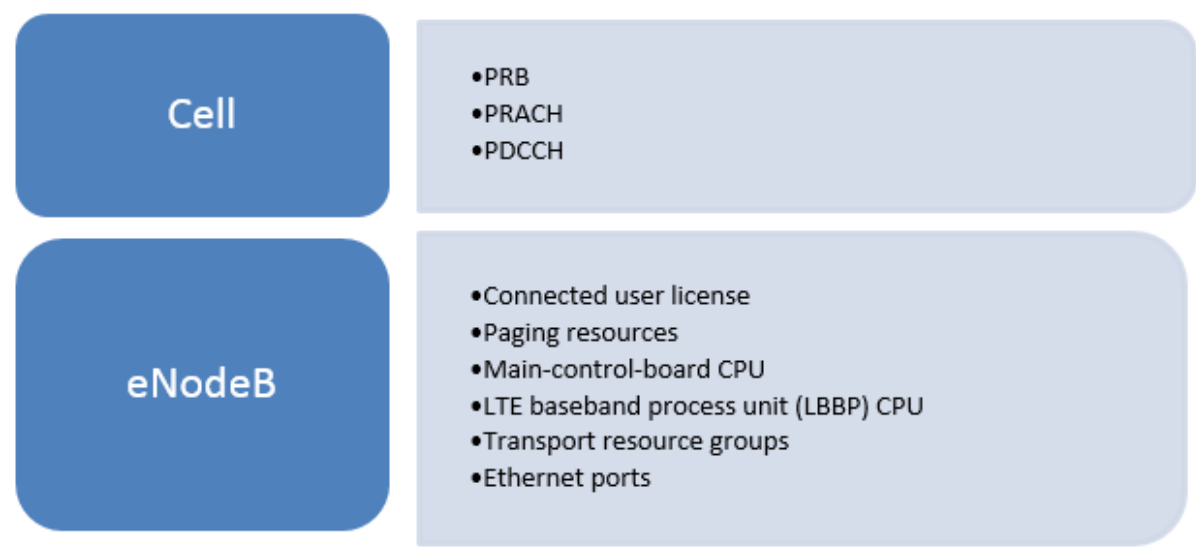

Fig. 1. LTE cell and eNodeB resources.

This paper concentrates on LTE Cell Resources.

\section{PRB: Physical Resource Block}

Resource Block is the minimum unit for resource allocation used for data transmission in physical layer, it consists of two dimensional domain: [3]

- Time Domain: 1 Slot= 7 OFDM Symbol (normal CP).

- Frequency Domain: 12 Subcarriers.

RB number depend on the LTE system bandwidth as shown in table 1

Table 1. RB number vs LTE system bandwidth.

\begin{tabular}{|c|c|c|c|c|c|c|}
\hline Channel bandwidth BW $_{\text {Channel }}[\mathrm{MHz}]$ & 1 & 3 & 5 & 10 & 15 & 20 \\
\hline Sub-carriers Number & 72 & 180 & 300 & 600 & 900 & 1200 \\
\hline RB Number & 6 & 15 & 25 & 50 & 75 & 100 \\
\hline
\end{tabular}

\subsection{Resource Element (RE)}

Smallest unit in PRB consist of two dimensional domain:

- Time Domain: 1 OFDM Symbol.

- Frequency Domain: 1 Subcarriers. 


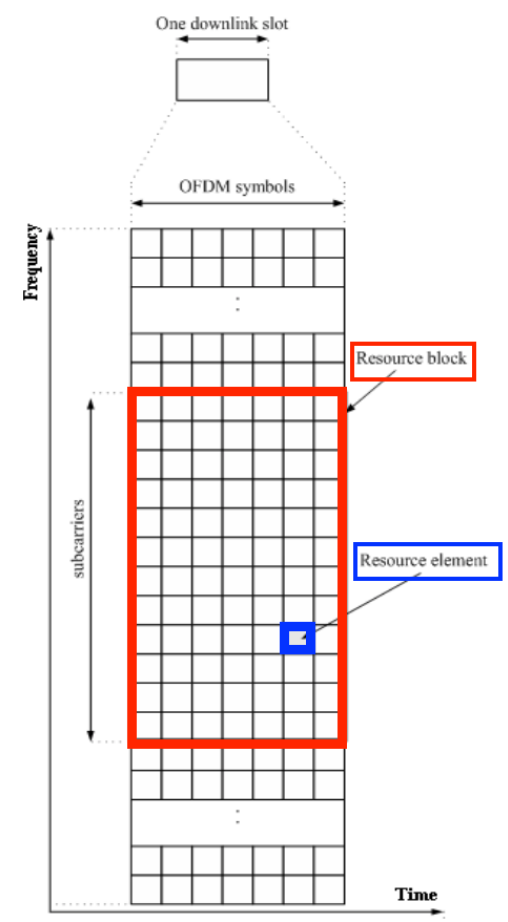

Fig. 2. LTE resource element.

\subsection{Usage Increase by}

- As Data traffic growth PRB Usage increases.

- As PRB Usage reaches $100 \%$, user perceived traffic will decrease.

\subsection{Insufficiency}

- Growing traffic leads to a continuous increase in PRB usage. When the PRB usage approaches to $100 \%$, user-perceived rates will decrease as follow:

- New Users may fail to be admitted

- Admitted users experience is affected.

\subsection{Monitoring}

- Downlink PRB usage = L.ChMeas.PRB.DL.Used.Avg/L.ChMeas.PRB.DL.Avail x $100 \%$

- L.ChMeas.PRB.DL.Used.Avg = Average used DL PRBs

- L.ChMeas.PRB.DL.Avail = Available DL PRBs

- Downlink user-perceived rate (Mbit/s) = L.Thrp.bits.DL/L.Thrp.Time.DL/1000

- L.Thrp.bits.DL = DL PDCP Layer Throughput 
- L.Thrp.Time.DL= duration for transmitting DL PDCP layer data

\subsection{Root Cause Analysis/Solution}

- Factors affecting PRB usage:

- Poor Channel Quality

- Parameter Settings

- User number

- Traffic Model "service requirement of each user"

- PRB Limitation leads to prolong UE Scheduling delay $\rightarrow$ decreasing UE Data Rate.

\subsection{Poor Channel Quality}

- Lead to low spectrum efficiency and increase PRB usage.

- DL Spectrum Efficiency = L.Thrp.bits.DL/L.ChMeas.PRB.DL.Used.Avg/ (rep rt Period*60)/180000 "bit/s/Hz"

- If Less than $1 \mathrm{bit} / \mathrm{s} / \mathrm{Hz} \rightarrow$ Optimize RF Quality

- UL Spectrum Efficiency = L.Thrp.bits.UL/L.ChMeas.PRB.UL.Used.Avg/ (report Period*60)/180000 "bit/s/Hz"

- If Less than $0.5 \mathrm{bit} / \mathrm{s} / \mathrm{Hz} \rightarrow$ Optimize RF Quality

\subsection{Parameter Settings}

- DL

- RbgAllocStrategy=Adaptive

- If the number of required RBs is less than that of one RBG, RBs are allocated to the scheduled UE as required, which is specified by resource allocation type 1.

- If the number of required RBs is greater than that of one RBG, the number of allocated RBGs is rounded up and an integral number of RBGs are allocated to the scheduled UE. Compared with RBG round-up, this mode prevents RB waste when the number of required RBs is less than that of one RBG

- UL

- UlSchSwitch=PuschDtxSwitch-1

- During UL transmission if UE fail to detect UL GRANT or SR false alarm appear in DRX state

- Enabled:UE will report(ACK/NACK/DTX) if the HARQ feedback state of one uplink schedule is DTX, eNodeB will deliver UL GRANT again instead of starting HARQ retransmission. If the next HARQ feedback is still DTX, eNodeB will stop the schedule triggered by this SR

- Disabled:lead to the uplink retransmission repeatedly and waste the uplink PUSCH resource and the uplink error code rate will increase obviously. 


\subsection{User Number}

- If average RRC_Connected User(L.Traffic.User.Avg) $>300$

- Downlink user-perceived rate $<$ a user-defined threshold (default value: $2 \mathrm{Mbit} / \mathrm{s}$ )

- Resource Insufficiency cause the low Data Rate $\rightarrow$ Consider Expansion

- Formula for Downlink user perceived rate:
- Average
DL
Throughput
for single User
User $\quad=$

(L.Thrp.bits.DLL.Thrp.bits.DL.LastTTI)/L.Thrp.Time.DL.RmvLastTTI*1000

\subsection{Threshold for Expansion}

- Add carriers or eNodeBs if both of the following conditions are met:

- Downlink PRB usage $\geq 70 \%$

- Downlink user-perceived rate $<$ a user-defined threshold (default value: $2 \mathrm{Mbit} / \mathrm{s}$ )

\subsection{OMSTAR Analysis: Operation \& Maintenance}

- Whole Network PRB Analysis

- PRB Resource Usage Distribution

- Uplink/Downlink PRB Resource Usage Distribution (RRU)

- The Cell List of Uplink/Downlink PRB Resource Usage Over Threshold

- PRB Resource Usage(Daily)(Cell/Busy Hour)(Network/Busy Hour)

\subsection{UL / DL Formulas}

- Uplink PRB resource usage = Average number of uplink PRBs scheduled in each second

- (L.ChMeas.PRB.UL.Used.Avg+ L.ChMeas.PRB.PUCCH.Avg)/ Total number of uplink PRBs in a cell x 100\%

- Downlink PRB resource usage $=$ Average number of downlink PRBs scheduled in each second

- (L.ChMeas.PRB.DL.Used.Avg)/ Total number of downlink PRBs in a cell x 100\%

\subsection{OMSTAR Threshold is}

- OMSTAR threshold for Expansion is 70\% PRB usage.

Table 2. PRB cell usage KPIs.

\begin{tabular}{|c|c|c|c|c|}
\hline Time & $\begin{array}{c}\text { Whole Network } \\
\text { Average Number } \\
\text { of Uplink Used PRB } \\
\text { per Cell }\end{array}$ & $\begin{array}{c}\text { Whole Network } \\
\text { Average Number } \\
\text { of Downlink Used } \\
\text { PRB per Cell }\end{array}$ & $\begin{array}{c}\text { Whole Network } \\
\text { Average Uplink } \\
\text { PRB Resource } \\
\text { Usage per Cell }\end{array}$ & $\begin{array}{c}\text { Whole Network } \\
\text { Average Downlink } \\
\text { PRB Resource } \\
\text { Usage per Cell }\end{array}$ \\
\hline$x$ & 4.33 & 4.85 & $8.65 \%$ & $9.71 \%$ \\
\hline
\end{tabular}




\subsection{For Low Spectral Efficiency}

DL (1bPs/Hz):

Check if $\mathrm{MCS}<10$ or $\mathrm{CQI}<6 \rightarrow$ RF Issues $\rightarrow$ otherwise Check MIMO Setting

$\mathrm{UL}(0.5 \mathrm{bps} / \mathrm{HZ})$ :

Check if $\mathrm{MCS}<10$ or $\mathrm{SINR}<5 \rightarrow$ RF Issues

Downlink/Uplink Spectral Efficiency (Daily) (Cell/BH) (Network/BH)

\section{PRACH: Physical Random Access CHannel}

Random access is performed in the following scenarios: [3]

1. Initial RRC connection setup to switch from the RRC_IDLE state to the RRC_CONNECTED state, a UE initiates random access.

2. $\mathrm{RRC}^{-}$connection reestablishment When a radio link failure (RLF) occurs, the UE needs to reestablish an RRC connection. In this scenario, the UE initiates random access.

3. Handover During a handover, a UE initiates random access in the target cell.

4. Downlink data arrival when an eNodeB needs to send downlink data to a UE in the RRC_CONNECTED state and finds that the UE is out of uplink synchronization, the $\overline{\text { NN}}$ odeB instructs the UE to initiate random access.

5. Uplink data arrival When a UE in the RRC CONNECTED state needs to send uplink data to an eNodeB and finds that it is out of uplink synchronization, the UE initiates random access.

6. LCS When a location service (LCS) is required, the eNodeB initiates random access.

\subsection{Random Access message}

As shown in below figure consist of

- CP "Cyclic prefix"

- preamble sequence

- Guard period

Since Random Access message is first message sent from UE side and the TA "Time advance" is not known yet, so guard period is used to ensure message arrival is within the TS.

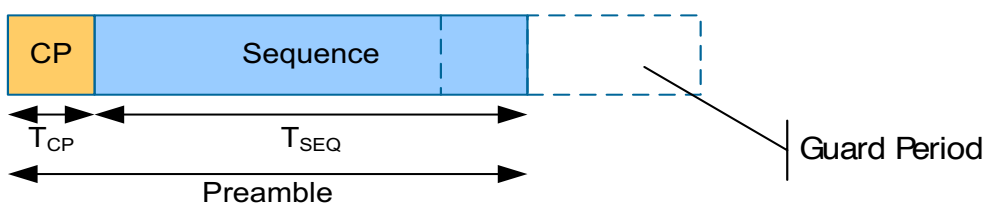

Fig. 3. Random Access message 


\subsection{Usage Increase by}

- Access requests (initial- UL/DL Data).

- Handover times.

- LCS

- RRC connection reestablishment.

\subsection{Root Cause analysis/Solution}

Enable Random Access Backoff. The RACH does not interfere with other uplink channels in LTE. Generally, the probability of RACH conflicts is low.

If excessive UEs are admitted on a PRACH, however, preamble conflicts may occur, and some UEs may fail to access the network. To solve this problem, random access backoff is introduced to control the time for preamble retransmission by UEs. [4]

If random access backoff is enabled, the eNodeB notifies a UE of a backoff value in the random access response message. When the UE needs to retransmit a preamble, it randomly selects a value (from 0 to the received backoff value) as its backoff time. The UE can retransmit the preamble only after the backoff time ends.

Random access backoff is not performed in the following two scenarios:

- Initial preamble transmission

- Preamble retransmission in non-contention-based random access

When to use?

If the random preamble usage reaches or exceeds $75 \%$ for three days by default in a week, enable the adaptive backoff function.

PRACH resource adjustment algorithm. In case of Preambles are insufficient PRACH resource adjustment algorithm acts as follow:

If dedicated preambles are in surplus and random preambles are insufficient, the eNodeB reduces the number of dedicated preambles.

If dedicated preambles are insufficient, the eNodeB increases the number of dedicated preambles.

Whether dedicated preambles are insufficient is measured based on the dedicated preamble allocation failure rate, which is equal to one minus the ratio of the number of UEs that are allocated dedicated preambles to the number of UEs that apply for dedicated preambles.

MaksIdxSwitch: Indicates the switch used to control reuse of dedicated preambles between UEs. If the switch is on, the eNodeB enables reuse of dedicated preambles among UEs based on the MaskIndex parameter. If the switch is off, the eNodeB allocates a dedicated preamble to only one UE at a time.

When to use?

If the dedicated preamble usage reaches or exceeds $75 \%$ for $\mathrm{X}$ days (three days by default) in a week, enable the PRACH resource adjustment algorithm and reuse of dedicated preambles between UEs. 
This helps reduce the probability of UEs initiating contention-based random access in the case of dedicated preamble insufficiency and therefore helps reduce the access delay.

Threshold for Expansion. If above actions implemented and the resource usage is still $>75 \%$ add eNodeB or split Cell.

\section{Profit from LTE RF Capacity Management and Optimization}

By monitoring, managing and optimizing LTE Capacity you can:

- Avoid Access Failure

- Improve UE Experience and main 4G/4.5G/5G KPIs

- Decrease Handover Delay

- Decrease Uplink and downlink scheduling delays.

\section{Conclusion}

This paper provides an overview of LTE capacity optimization steps and provide a new approach for $4.5 \mathrm{G} / 5 \mathrm{G}$ three main KPIs improvement (massive connections, low latency and high throughput). This article discusses also RF optimization solutions of LTE cell resource. Our analysis results by OMSTAR Tool show that the targets can be achieved in all LTE expansion scenarios to $4.5 \mathrm{G}$ and $5 \mathrm{G} / \mathrm{IoT}$.

\section{Acknowledgment}

This paper is supported by Huawei and Ericsson, 5G/IoT Tech Lab, Maroc Telecom R\&D Center.

\section{$7 \quad$ References}

[1] 4.5G, Opening Giga Mobile World, Empowering Vertical Markets, Huawei, white paper, 2015.

[2] ICT-317669 METIS project, Updated scenarios, requirements and KPIs for 5G mobile and wireless system with recommendations for future investigations, Deliverable D1.5, April 2015.

[3] G. Gomez et al., "Towards a QoE-Driven Resource Control in LTE and LTE-A Networks," J. Computer Networks and Commun., 2013.

[4] R1-157647, "Summary of evaluation results", Huawei, HiSilicon, 


\section{Authors}

Dr. Ahmed EL MAHJOUBI received state engineer degree in networks \& telecommunication systems from the National School of Applied Sciences Kenitra, Morocco, in 2014. Since 2014 he has been a Technical Director in the Department of Network Performances Service (NPS), Huawei Technologies. His main research interests include 3G/4G/5G systems QoS/QoE/KPIs, Network Audit, Network Planning and Optimization, wireless communication systems especially in the topics of RF systems.

He has been involved in several national and international MBB projects on RF Systems: IAM 2G/3G SingleRAN Project, IAM LTE \& VoLTE Project, IAM GUL Project, INWI 3G/4G Project, INWI UMTS900 Project and Bouygues Telecom 4G/5G Project. He has been the radio coordinator of the first test of 4G+ in Morocco \& Africa.

Prof. Tomader Mazri, HDR degree in Networks and Telecommunication from IbnTofail University, Ph.D. degree in Microelectronics and Telecommunication from SidiMohamed BenAbdellah University and INPT of Rabat, Master's degree in Microelectronics and Telecommunication Systems, Bachelor's degree in telecommunication from CadiAyyad university. She is currently a professor at National School of Applied Sciences of Kenitra and a Permanent member of Electrical and Telecommunications Engineering Laboratory. Author and co-author of twenty articles journals, forty articles in international conferences, a chapter and three books. Her major research interests are on Microwave systems for mobile and radar, Smart antennas and NG Mobile network.

Prof Nabil HMINA, Professor of Higher Education Director of the National School of Applied Sciences Kenitra, since November 2011 to date, Degree in Physics, Option: Thermodynamics - Mohammed V University, University PhD - Engineering Sciences, University and Ecole Centrale de Nantes, 1994, HDR (1st in Morocco) Ibn Tofail University, Kenitra, 2002. Vice-President for Academic Affairs and Information Technology of the University Ibn Tofail, 2005-2011 Post-Doctoral Researcher: EDF - thermokinetics Laboratory of Nantes, 1994-1995, Research Engineer at PolyTech school of Nantes, 1995-1997. Director of the research laboratory "Systems Engineering" since 2012 (15 permanent teachers 10 teachers Researchers Associate researchers $75 \mathrm{PhD}$ students.

Author and/or co-author of a dozen articles in notorious newspapers and forty papers in international conferences. Jury President, member of several theses and Habilitations for research orientation.

Article submitted 21 July 2018. Resubmitted 20 October 2017. Final acceptance 21 October 2018. Final version published as submitted by ther authors. 